\title{
Simulations of geometry effects and loss mechanisms affecting the photon collection in photovoltaic fluorescent collectors
}

\author{
L. Prönneke ${ }^{1, a}$, G.C. Gläser ${ }^{1}$, and U. Rau ${ }^{2}$ \\ 1 Institut für Photovoltaik, Universität Stuttgart, Pfaffenwaldring 47, 70569 Stuttgart, Germany \\ 2 IEK5-Photovoltaik, Forschungszentrum Jülich, 52425 Jülich, Germany
}

Received: 29 August 2011 / Accepted: 29 February 2012

Published online: 26 June 2012

\begin{abstract}
Monte-Carlo simulations analyze the photon collection in photovoltaic systems with fluorescent collectors. We compare two collector geometries: the classical setup with solar cells mounted at each collector side and solar cells covering the collector back surface. For small ratios of collector length and thickness, the collection probability of photons is equally high in systems with solar cells mounted on the sides or at the bottom of the collector. We apply a photonic band stop filter acting as an energy selective filter which prevents photons emitted by the dye from leaving the collector. We find that the application of such a filter allows covering only $1 \%$ of the collector side or bottom area with solar cells. Furthermore, we compare ideal systems in their radiative limits to systems with included loss mechanisms in the dye, at the mirror, or the photonic filter. Examining loss mechanisms in photovoltaic systems with fluorescent collectors enables us to estimate quality limitations of the used materials and components.
\end{abstract}

\section{Introduction}

Fluorescent collectors (FCs) use organic dye molecules or inorganic fluorescent quantum dots surrounded by a dielectric material to trap and concentrate solar photons. The dye absorbs incoming photons with energy $E_{1}$ and emits photons due to Stokes shift with $E_{2}<E_{1}$. The emission occurs with a randomized direction. Total internal reflection traps part of the radiation in the system and guides the photons to the collector sides. In a photovoltaic system, solar cells applied to the collector sides or the back side collect these photons and convert them into electrical energy. Already in the late 1970s and early 1980s Goetzberger, Wittwer and Greubel described the technological potential of FCs in photovoltaic systems [1,2]. Recently, the basic idea has regained some interest in the context of building photovoltaic structures which exceed the classical efficiency limitations by using up- and downconverting dyes $[3-5]$. Theoretical tools to describe FCs thermodynamically have been developed [6-8]. Numerical approaches analyzing the FC behavior gain more interest $[9,10]$. In order to estimate theoretical limitations, the photovoltaic systems with FCs have been highly idealized. However, realistic setups show loss mechanisms which need to be considered. The classic idea of assembling an FC in a photovoltaic system is based on its behavior of guiding emitted photons to the sides. Therefore, the classical setup mounts solar cells to the collector sides [2,11-17].

${ }^{a}$ e-mail: liv.proenneke@ipv.uni-stuttgart.de
Technically, it seems less expensive to apply and connect solar cells at the bottom side of the collector. Experimentally, fluorescent collectors and photonic structures on top of solar cells prove to raise the output current by $95 \%$ compared to a non-fluorescent glass on top of the cell [18].

The present paper uses Monte-Carlo ray-tracing simulations for a comparison of the classical side-mounted system to a system where solar cells cover the FC back side. We see that the side-mounted system performs better in most cases, especially at larger collector sizes. However, for both systems the maximum collection probability for photons $p_{c}=97 \%$ is only achieved in the presence of a back side mirror and a photonic band stop (PBS) filter at the collector top surface acting as an energy selective filter. This maximal photon collection occurs in the statistical limit. Here, numerous small solar cells cover the FC in close proximity. Such a small-scale system is more favorable than a system with few large-scaled solar cells taking the same coverage fraction. The maximal number of collected photons is equivalent in a photovoltaic system with neither FC nor PBS, but our assembly saves us $99 \%$ of solar cell area.

In order to describe FCs in photovoltaic systems, we use numerical and analytical approaches based on the principle of detailed balance. Starting from ideal systems in their radiative recombination limitation, we also examine the influences of non-radiative recombination in the fluorescent dye, of non-perfect reflection at the mirrors, and of non-perfect reflection conditions at the PBS filter. The results point out that reflection losses at the back 


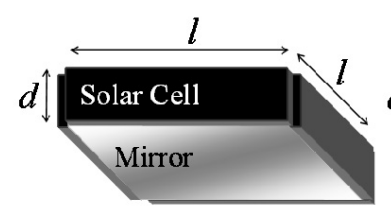

(a)

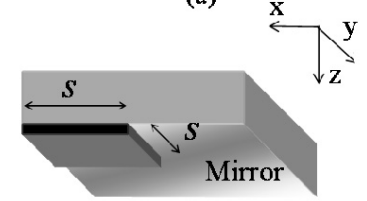

(c)

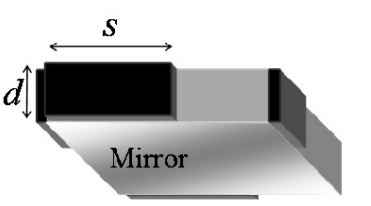

(b)

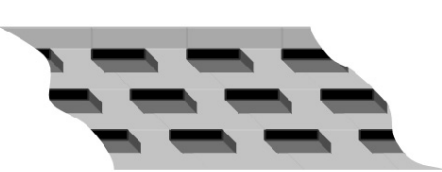

(d)
Fig. 1. Sketch of the fluorescent collector geometries compared in the present paper (seen from the bottom). (a) Classical design with the solar cells mounted at each side of the collector with length $l$ and thickness $d$. (b) Modified classical system where only a fraction of the respective collector sides is covered with a solar cell area $A_{\text {cell }}=d s$. (c) Collector with solar cells with an area $A_{\text {cell }}=s^{2}$ mounted at the bottom. In all cases the (remaining) back side is covered with a mirror. (d) Systems (b) and (c) are assumed to be periodic in space. Exemplary, a detail of the bottom-mounted system is shown.

surface cause a higher decrease than losses due to nonradiative recombination in the dye. Compared to a system without applied PBS non-radiative losses induce higher decreases of photon collection in the PBS covered system.

\section{Collector geometries and dye properties}

This section describes the three photovoltaic systems discussed in this paper. A characterization of the FC dye properties as well as an explanation of the functionality of a PBS filter follows.

Figure 1a shows an FC in the classical configuration with an acrylic plate of length $l$ and thickness $d$ doped with fluorescent dye. The collecting solar cells are mounted at the sides of the plate. Let us define the coverage fraction $f=A_{\text {cell }} / A_{\text {coll }}$ as the ratio between the area $A_{\text {cell }}=4 d l$ of the solar cells in the system and the illuminated collector area $A_{\text {coll }}=l^{2}$. For the configuration in Figure 1a, we have $f=4 d l / l^{2}=4 d / l$, hence the coverage fraction depends only on the ratio between the collector thickness $d$ and the side length $l$. A perfect mirror covers the FC back side. Figure $1 \mathrm{~b}$ features a variant of the side-mounted FC where only a part of each side is covered with a solar cell. The system is repeated periodically in $x$ - and $y$-direction. Therefore, photons hitting a collector side experience periodic boundary conditions and enter the opposite side. In an alternative but equivalent perception perfect mirrors cover the remainders. The coverage fraction for the system in Figure $1 \mathrm{~b}$ is $f=4 d s / l^{2}$ with the side length $s \leq l$ of the solar cells. Thus, coverage fraction $f$ and collector length $l$ are decoupled and this geometry offers an additional degree of freedom for the collector design. Again, the FC back side is covered by a mirror. The collector design of Figure 1c uses a square solar cell with a side length $s$ at the back side of the FC. Thus, the solar cells in this

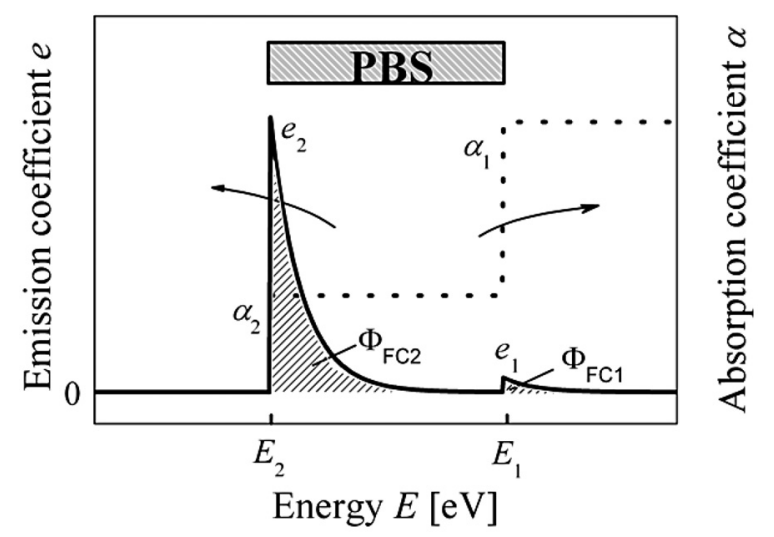

Fig. 2. Sketch of the absorption and emission behavior as assumed in this paper. The dye absorption is given by a step function. Incoming photons have the energy $E_{1}$ and a high absorption coefficient $\alpha_{1}$. The lower absorption coefficient $\alpha_{2}$ holds for the lower energy $E_{2}$ and leads with Kirchhoffs law (Eq. (1)) to a high emission coefficient $e_{2}$. The model also features the possibility of an energy selective photonic band stop (PBS) that keeps the emitted photons in the FC system.

bottom-mounted system cover a fraction $f=s^{2} / l^{2}$ of the surface. Figure 1d shows a detail of the bottom-mounted system which is also assumed to be periodically repeated in $x$ - and $y$-direction. As shown, square solar cells occupy the back surface of the collector with a period length $l$. The remaining parts of the back side are covered with a mirror.

We model an FC consisting of an acrylic plate with the refractive index $n_{r}=1.5$ and embedded fluorescent dye molecules. Figure 2 depicts the absorption/emission behavior of the fluorescent dye used in the following. We assume a stepwise increase of the absorption constant $\alpha$ from zero at energies $E<E_{2}$ to a value $\alpha_{2}$ for $E>E_{2}$ and a further increase to $\alpha_{1}$ for energies $E>E_{1}$. The emission coefficient $e$ is linked to the absorption coefficient $\alpha$ via Kirchhoffs law

$$
e(E)=\alpha(E) n_{r} \phi_{b b}(E)
$$

with the black body spectrum

$$
\phi_{b b}(E)=\frac{2}{h^{3} c^{2}} \frac{E^{2}}{e^{E / k T}-1} \approx \frac{2 E^{2}}{h^{3} c^{2}} e^{-E / k T}
$$

where $n_{r}$ is the refractive index of the collector material, $h$ is Planck's constant, $c$ the speed of light, and $k T$ the thermal energy corresponding to the temperature $T$ of the collector and its surroundings ( $T=300 \mathrm{~K}$, throughout this paper).

The absorption/emission dynamics used in the following is given by a two-level scheme as used earlier to describe the detailed balance limit of FCs $[11,12,19]$. The choice of this simple approach ensures a certain generality of our results such that the trends caused by the collector geometries or by the introduction of loss mechanisms should be equally found in real systems with more complex 
spectral absorption/emission properties. For the present two-level system we consider the emission probabilities

$$
p_{1}=\frac{\alpha_{1}}{p} \int_{E_{1}}^{\infty} E^{2} \exp \left(-\frac{E}{k T}\right) d E=\frac{\alpha_{1} p_{\infty}\left(E_{1}\right)}{p}
$$

and

$p_{2}=\frac{\alpha_{2}}{p} \int_{E_{2}}^{E_{1}} E^{2} \exp \left(-\frac{E}{k T}\right) d E=\frac{\alpha_{2}\left[p_{\infty}\left(E_{2}\right)-p_{\infty}\left(E_{1}\right)\right]}{p}$

for photon emission by the fluorescent dye in the range of photon energies $E>E_{1}$ and $E_{1}>E>E_{2}$, respectively. In equations (3) and (4), we use the definition

$$
\begin{aligned}
p_{\infty}\left(E_{x}\right) & =\int_{E x}^{\infty_{1}} E^{2} \exp \left(-\frac{E}{k T}\right) d E \\
& =k T\left[2(k T)^{2}+2 E_{x} k T+E_{x}^{2}\right] \exp \left(-\frac{E}{k T}\right)
\end{aligned}
$$

and the normalization factor $p$ such that $p_{1}+p_{2}=1$.

The choice of the energies $E_{1}, E_{2}$, and the absorption coefficients $\alpha_{1}, \alpha_{2}$ leads to the emission probabilities $p_{2} \gg$ $p_{1}$, in contrast to the absorption coefficients $\alpha_{1} \gg \alpha_{2}$. Due to the dominance of the exponential factor in equation (5) we approximate

$$
\frac{p_{1}}{p_{2}} \approx \frac{\alpha_{1}}{\alpha_{2}} \exp \left(\frac{E_{2}-E_{1}}{k T}\right) .
$$

Thus, a choice of an energy difference $\Delta E=E_{1}-E_{2}=$ $200 \mathrm{meV}$ and of absorption coefficients $\alpha_{1}=100 \alpha_{2}$ still ensures $p_{2} \approx 20 p_{1}$. In the following, we assume $E_{1}=2.0 \mathrm{eV}$, $E_{2}=1.8 \mathrm{eV}$ and absorption coefficients $\alpha_{1}=3 / d$, $\alpha_{2}=0.03 / d$. Therefore, the system provides a high emission coefficient $e_{2}$ for photons with energy $E_{2}$ and a significantly lower emission coefficient $e_{1}$ for photons with high energies.

Figures $3 \mathrm{a}-3 \mathrm{e}$ sketch the functionality of the PBS filter. Incoming photons have the energy $E_{1}$. The dye absorbs these photons and emits spatially randomized photons with angles $\theta, \phi$ defined in Figure $3 \mathrm{a}$ at a lower energy $E_{2}$. Figure $3 \mathrm{~b}$ shows that emitted photons impinging at the top surface with an incident angle $\theta$ higher than the critical angle $\theta_{c}$ for total internal reflection are guided to the collector sides. Whereas photons with $\theta<\theta_{c}$ leave the collector as shown in Figure 3c. The application of a PBS filter avoids this loss mechanism. Figure 3d shows the ideal PBS filter which is energy selective only $\left(\theta_{p b s}=\theta_{c}\right)$. The filter has a reflection $R=1$ and a transmission $T=0$ for photon energies $E<E_{t h}$. For the other part of the spectrum $R=0$ and $T=1$ is assumed. Therefore, $E_{t h}$ denotes the upper cut-off energy of the filter. We choose $E_{t h}=E_{1}$ throughout this paper. Two- and three-dimensional photonic crystals [20-22] are promising materials which might be used as omnidirectional PBS

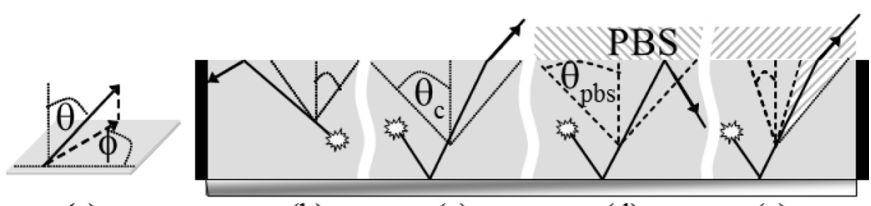

(a)

(b)

(c)

(d)

(e)

Fig. 3. Light guiding behavior of a fluorescent collector covered with solar cells at the sides and a mirror at its back side. (a) Definition of photon ray angle $\theta$. (b) Absorbed photons are reemitted spatially randomized. The system leads rays with $\theta>\theta_{c}$ to the sides of the collector. (c) Rays with angle $\theta<\theta_{c}$ for total internal reflection leave the top surface. (d) Applying a photonic band structure (PBS) keeps rays with $\theta<\theta_{p b s}$ in the system as well. This PBS is energy selective with $\theta_{p b s}=\theta_{c}$. Therefore, rays with energies $E \leq E_{1}$ are kept in the system only. (e) For an energy and angular selective PBS a reflection cone is assumed such that only rays with $E \leq E_{1}$ and $\theta<\theta_{p b s}$ are kept in the system.

in FC systems. However, technological developments have led to dielectric mirrors used as band pass filters with almost rectangular cut-off characteristics for normal incident photons $[23,24]$. These rugate-filters show a high angular dependency by blocking only photons with almost perpendicular incidence. In order to examine the influence of this angular selectivity, we vary the reflection cone of the filter. Figure $3 \mathrm{e}$ depicts that a PBS with $\theta_{p b s}<\theta_{c}$ reflects photons with $E \leq E_{1}$ and $\theta<\theta_{p b s}$. Thus, rays with $\theta_{c}>\theta>\theta_{p b s}$ hitting the collector surface within the striped angle cone are neither reflected by the PBS nor subject to total internal reflection and leave the system.

\section{Simulation method}

The Monte-Carlo simulation calculates the collection probability for photons $p_{c}$ for the different collector geometries shown in Figures $1 \mathrm{a}-1 \mathrm{c}$ with varied collector dimensions and component quality. In order to allow also the comparison between systems with and without PBS, we provide only incoming photons with energy $E=E_{1}$. All photons enter into the collector perpendicular with random coordinates $(x, y)$ with $0<x<l$ and $0<y<l$. Their statistical absorption occurs following Beer's absorption law after a path length

$$
w=-\frac{1}{\alpha_{1}} \ln \left(p_{w}\right)
$$

where $p_{w}$ is a random number $0 \leq p_{w} \leq 1$. After its absorption a photon is re-emitted with a probability $p_{e}=1-p_{n r}$ with the non-radiative recombination probability $p_{n r}$ of the fluorescent dye. According to equations (3) and (4) the energy of the re-emitted photons lies with the probability $p_{1}$ in the energy range $E \geq E_{1}$ and with $p_{2}$ in the range $E_{1}>E \geq E_{2}$. After re-emission the photon also obtains a pair of spherical angles $(\theta, \phi)$ with $0<\theta<\pi$ and $0<\phi<2 \pi$ using the probabilities $p_{\theta}=\sin (\theta) / 2$ and $p_{\phi}=1 / 2 \pi$ (for the definition of $\theta$ and $\phi$, see Fig. 3a). 
Subsequently, either the dye molecules reabsorb the re-emitted radiation or the photons hit one of the six collector surfaces at a coordinate $\left(x_{s}, y_{s}, z_{s}\right)$. At the top surface $\left(x_{s}, y_{s}, 0\right)$, the photon is reflected if $\theta>\theta_{C}$ with $\sin \left(\theta_{C}\right)=1 / n_{r}$. In the presence of an omnidirectional PBS, the photon is reflected for all photon energies $E<E_{1}$. An assumed angular selectivity sets as a reflection condition $E<E_{1}$ and $\theta<\theta_{p b s}$ as defined in Figure 3e. Non-reflected photons are lost and the number $N_{\text {lost }}$ of lost photons is increased accordingly. At the bottom surface $\left(x_{s}, y_{s}, d\right)$ a mirror perfectly reflects the photons with a probability $p_{r}=1$. We use $p_{r}<1$ for the analysis of loss mechanisms. For the system shown in Figure 1c, the bottom-mounted solar cells collect photons with $x_{s} \leq s$ and $y_{s} \leq s$. In this case, the photons add to the number $N_{\text {coll }}$ of collected photons. A special case discussed below is the statistical limit where we simply assume that a photon hitting the bottom of the collector enters a solar cell with the probability $f$, the solar cell coverage fraction. Throughout this paper, we assume a collection probability of $100 \%$ for photons hitting the solar cell area with energy $E$ higher than the solar cell band gap $E_{\text {gap }}$. In order to analyze the principle limitations of applying FCs to photovoltaic systems, we choose $E_{g a p}=1.8 \mathrm{eV}$ which corresponds to the emission peak of the FC.

If photons hit the collector sides, for instance at $\left(l, y_{s}, z_{s}\right)$ for the right collector side, side-mounted solar cells collect the photons for the geometry shown in Figure 1a. The geometry depicted in Figure 1b collects photons hitting the collector right surface with $y_{s} \leq s$. Otherwise, the photon is subject to periodic boundary conditions. For the bottom-mounted system, we apply periodic boundary conditions on each collector side, i.e. the photon is re-injected at the respective facing side with unchanged spherical angles $(\theta, \phi)$.

Our ray tracing program, that typically handles a number $N_{\text {in }}=5 \times 10^{4}$ photons in parallel, runs until all photons are collected by the solar cells, lost by re-emission from the collector surface, by non-radiative recombination in the dye, by reflection losses at the mirrors or at the PBS. With $N_{\text {in }}=N_{\text {lost }}+N_{\text {coll }}$, we obtain the collection probability for photons $p_{c}=N_{\text {coll }} / N_{\text {in }}$ as the final result.

\section{Simulation results}

In this section, we describe the simulation results. First, the classical FC system with side-mounted solar cells as shown in Figure 1a is modeled. Here, the variation of the collector dimensions modifies the coverage fraction of solar cells. Second, photon collection probabilities for the bottom-mounted system as depicted in Figure 1c are calculated. In these simulations, we already include the loss mechanism of non-radiative recombination in the dye. The influences of collector dimension and coverage fraction are de-coupled in this system. In the third part, the approach of de-coupling these two aspects also in the side-mounted system as sketched in Figure $1 \mathrm{~b}$ allows the comparison between side-mounted and bottom-mounted

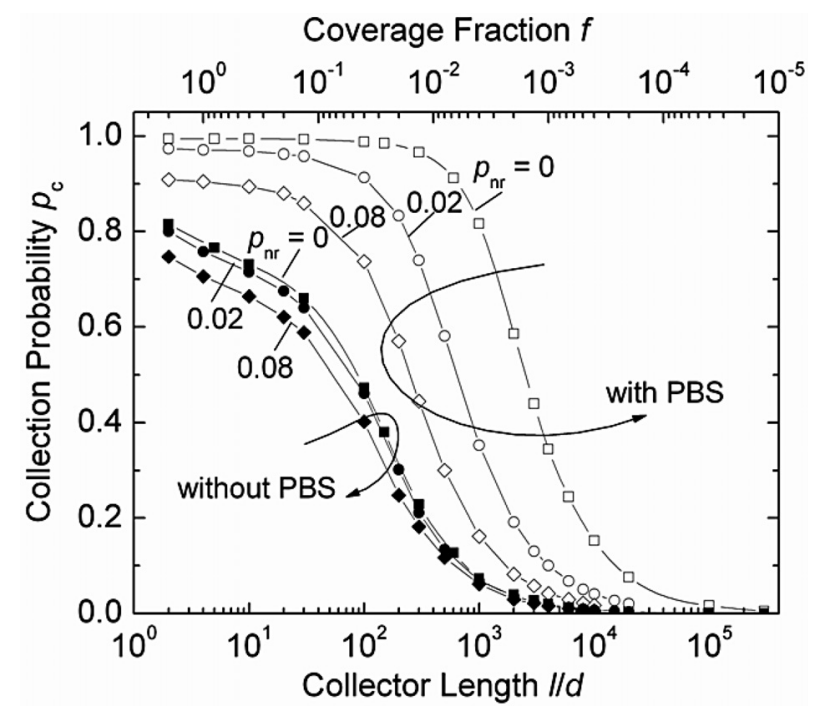

Fig. 4. The collection probability $p_{c}$ of the classical fluorescent collector geometry (Fig. 1a) fully covered with solar cells at its sides depends on the collector length $l$ normalized to the thickness $d$. A perfect mirror covers the back side. The systems with a photonic band stop filter (PBS, open symbols) have higher collection probabilities than those without PBS (filled symbols). For the system with PBS a value of $p_{c}$ close to unity remains up to a normalized collector length $l / d \approx 500$ and nonradiative recombination probability $p_{n r}=0$ in the dye. In contrast, the system without PBS has a maximum $p_{c}$ only slightly above $80 \%$ even in the radiative case with $p_{n r}=0$. With increasing collector length $l / d$ the collection probability $p_{c}$ decreases immediately. The consideration of non-radiative recombination in the dye, i.e. $p_{n r}>0$, leads to a deterioration of $p_{c}$ in all cases. The cell coverage fraction $f$ (top axis) is directly linked to the collector size for the chosen geometry.

system. We compare both systems, first at constant collector lengths for varied coverage fractions. Secondly, at constant coverage fractions under the inclusion of three loss mechanisms: a non-radiative recombination in the dye, a non-perfect mirror at the collector back side, and an angular selectivity at the photonic structure lying on top of the collector.

\subsection{Classical collector geometry}

This subsection studies the influence of the collector and cell geometry on the photon collection properties of a classical collector system as shown in Figure 1a. Figure 4 shows the dependence of the collection probability $p_{c}$ on the collector length $l / d$ normalized to the collector thickness $d$ calculated for systems with and without PBS at the top surface. We have also considered non-radiative recombination in the dye by assuming $p_{n r}=0.02$ and 0.08 .

The most important feature in Figure 4 is that the systems with PBS have a considerably higher collection probability $p_{c}$ than those without PBS. This is because the PBS decreases the emission of photons through the surface of the collector as shown in Figure 3c. For systems without PBS this non-radiative loss occurs whenever a 
photon falls into the critical angle $\theta_{c}$ of total reflectance. For the system with PBS the photon additionally must be emitted at an energy $E \geq E_{1}$. This emission probability is low, but non-zero for reasons of detailed balance. For the same reason systems with PBS obtain the high values of $p_{c}$ also for larger collector lengths $l / d$, whereas for the systems without PBS $p_{c}$ drops considerably already upon slight increases of $l / d$. Furthermore, with increased $l / d$ also the number of photons absorbed by the dye a second or third time increases. Each absorption event leads to $\theta$-randomization of the re-emitted photon and, in consequence, to a certain probability that the photon is lost by emission from the collector surface.

Also shown in Figure 4 are curves that reflect nonradiative recombination in the dye $\left(p_{n r}=0.02\right.$ and 0.08$)$. We see that the system with PBS is especially sensitive to non-radiative recombination in the range of large $l / d$. At low values of $l / d$ a photon is most likely absorbed only once before collected by the side-mounted solar cells. The maximum of $p_{c}$ decreases therefore only proportionally to $\left(1-p_{n r}\right)$. For higher values of $l / d$, repeated re-absorption of photons not only increases the risk for radiative but now also for non-radiative losses. As radiative losses are low in the systems with PBS the relative importance of non-radiative losses is higher. Whereas in the radiative case a value of $p_{c}>90 \%$ remains up to a normalized collector length $l / d \approx 500$, for $p_{n r}=0.02$ and 0.08 we have $p_{c}>90 \%$ only for $l / d \leq 100$ and $l / d \leq 6$, respectively. The changes that occur by non-radiative recombination in the case of systems without PBS over the whole range of $l / d$ are less significant due to the high emission losses that are present in the system anyway.

\subsection{Scaling effect}

The classical side-mounted collector geometry has a strict relation between the collector length $l$ and the cell coverage fraction $f=A_{\text {cell }} / A_{\text {coll }}=4 d / l$. In this subsection we examine the bottom-mounted system shown in Figure 1c where both quantities can be treated independently. For Figure 5 the coverage fraction $f=0.01$ is fixed and we vary the collector length $l$. Because of $f=s^{2} / l^{2}$, we adjust the cell side length $s$ to $s=l \sqrt{f}$.

Figure 5 demonstrates that the collection probability $p_{c}$ at constant coverage fraction $f$ drastically depends on the collector length $l / d$. The data in Figure 5 display asymptotic behavior in both limits, for small and large ratios $l / d$. We observe a wide transition regime between the two limiting cases where the collection probability $p_{c}$ changes from high values at small $l / d$ to significantly smaller values at large $l / d$. Such a behavior is typical for spatially extended inhomogeneous systems. If the characteristic feature length (here the collector length $l$ ) is large with respect to the length scale that is characteristic for interactions within the system (here the mean free path of photons), the system can be looked at as a parallel connection of spatially separated subsystems without interaction. The collection probability $p_{\mathrm{c}, \mathrm{ls}}$ in this large scale limit [25] is then the weighted average of a portion $f$ that

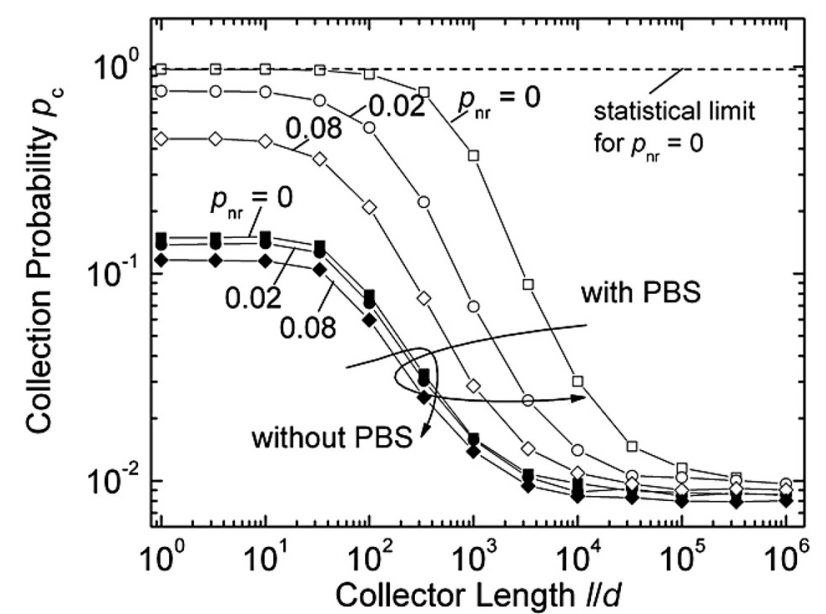

Fig. 5. Collection probability $p_{c}$ of the fluorescent collector geometry with solar cells mounted at the bottom of the collector (Fig. 1c). The coverage fraction $f$ is kept constant at $f=0.01$ and the normalized collector length $l / d$ is varied. All data feature a transition between two asymptotic situations at low and high ratios $l / d$. Only the systems with a photonic band pass (PBS, full symbols) achieve $p_{c}>0.5$ at low ratios of $l / d$. For these systems, the maximum $p_{c}$ as well as the transition from the small-scale to the large-scale behaviour is strongly dependent on the non-radiative recombination probability $p_{n r}$. The systems without PBS (open symbols) have already a relatively low $p_{c}<20 \%$ even in the more favorable case of $l / d<10$. Accordingly, the sensitivity to the introduction of non-radiative recombination in the dye $\left(p_{n r}>0\right)$ is less pronounced.

has a local $p_{c}$ of a collector with full back coverage, i.e. $p_{c} \approx 1$, for the system with $p_{n r}=0$ and a portion $(1-f)$ with $p_{c}=0$. This is why we observe in this limit that $p_{c}$ of the system with PBS approaches the coverage fraction $f$.

In contrast, approaching the small scale limit $(l<$ $1 / \alpha_{2}$, marked in Figure 5 with dashed line [26]), any ray, reflected forth and back within the collector, has often the possibility to hit a cell at the collector back side. In this situation, the system might be looked at as spatially homogeneous with statistical cell coverage at its back. In fact, the value of $p_{c, s s}$ in the small scale limit is consistent with quasi-one-dimensional computations that simulate the bottom-mounted solar cells by a probability $p_{c}=f$ for a photon to be collected by cells at the back side. Therefore, we denote this case also as the statistical limit.

The introduction of non-radiative recombination $\left(p_{n r}=0.02,0.08\right)$ in Figure 5 leads to a deterioration of the collection probability in all cases. As we have already seen in Figure 4, the system with PBS is much more sensitive to losses in the dye because of its overall high collection probability in the radiative limit. Especially important is the influence of a finite $p_{n r}$ on the transition from the small-scale to the large-scale limit. Whereas with $p_{n r}=0$ all systems with $l / d \leq 100$ yield the same high collection probability, the limit for $p_{n r}=0.08$ reduces to $l / d \leq 10$.

All data in Figure 5 represent the same coverage $f=$ 0.01 , i.e. the same amount of solar cell area per unit collector area. Nevertheless, the collection probability strongly 
depends on the chosen size of collector and solar cell. A proper scaling of these quantities is therefore necessary to tune the collection probability and, finally, the collector performance to its optimum.

\subsection{Statistical limit}

The following section derives an analytical description for the statistical limit marked in Figure 5 with the dashed line [27]. This analytical description only holds for the statistical limit in the radiative case and for systems with applied photonic structure. As discussed by Markvart [28] and Rau et al. [29], using photon fluxes only describes systems with equal chemical potential $\mu$ for the incoming photons. Applying a PBS equalizes $\mu$ because the absorption coefficient for all incoming photons is now $\alpha_{1}$. In the Monte-Carlo simulation, we excite the system with a monochromatic beam with $E=2.22 \mathrm{eV}$. The results fit the calculation of the analytical expression because both cases fulfill the condition of equalized $\mu$. In contrast, the system without PBS does not provide a spectrally equal absorption for all incoming photons leading to an inhomogeneous $\mu$. Additionally, photons experience a spatial inhomogeneity for systems beyond the statistical limit as depicted in the former section. This also leads to an inhomogeneous $\mu$. Considering these limitations, we compare in the following a side- and a bottom-mounted system in the statistical limit $(l / d=1)$ with applied PBS.

The Monte-Carlo simulation gives us the expressions for the photon flux incident on the collector surface

$$
\varphi_{\text {sun }}^{M C}=\frac{N_{\text {in }}}{A_{\text {coll }}}
$$

the photon flux which is absorbed in the collector by the solar cells

$$
\varphi_{F C}^{M C}=\frac{N_{\text {coll }}}{A_{\text {cell }}},
$$

and the photon flux leaving the collector without hitting a solar cell

$$
\varphi_{\text {out }}^{M C}=\frac{N_{\text {in }}-N_{\text {coll }}}{A_{\text {coll }}} .
$$

We propose that the ratio between the incident photon flux $\Phi_{\text {sun }}$ and the photon flux $\Phi_{F C}$ kept in the collector by total internal reflection gives the expression

$$
c_{T I R}^{\max }=\Phi_{F C} / \Phi_{\text {sun }}
$$

which is at the same time the maximum concentration of a concentrator based on total internal reflection only. A collector with a refractive index of $n=1.5$ yields a concentration $c_{T I R}=2.25$. Inside a $\mathrm{FC}$ with a dielectric material doped with a spectral shifting dye, the overall flux

$$
\Phi_{F C}=\Phi_{F C 1}+\Phi_{F C 2}
$$

is composed of two fluxes $\Phi_{F C 1}$ and $\Phi_{F C 2}$ as depicted in Figure 2. Here,

$$
c_{p, \max }=\left(\Phi_{F C 1}+\Phi_{F C 2}\right) / \Phi_{\text {sun }}
$$

denotes the maximum concentration inside the FC. By integrating over the corresponding sections on the energy axes, the analytical expressions for the two fluxes inside the collector

$$
\Phi_{F C 1}=\frac{2 n^{2}}{h^{3} c^{2}} \int_{E_{1}}^{\infty} E^{2} e^{-E / k T} d E
$$

and

$$
\Phi_{F C 2}=\frac{2 n^{2}}{h^{3} c^{2}} \int_{E_{2}}^{E_{1}} E^{2} e^{-E / k T} d E
$$

are derived. The PBS reflecting all photons with energy $E_{2}<E<E_{1}$ limits the incident photon flux

$$
\Phi_{\text {sun }}=\frac{2}{h^{3} c^{2}} \int_{E_{1}}^{\infty} E^{2} e^{-E / k T} d E .
$$

With equations (14) to (16) it is possible to calculate the maximum concentration analytically

$$
c_{p, \max }=n^{2} \frac{\left(E_{2}^{2}+2 k T E_{2}+2(k T)^{2}\right) e^{-E_{2} / k T}}{\left(E_{1}^{2}+2 k T E_{1}+2(k T)^{2}\right) e^{-E_{1} / k T}}=4251
$$

with $k T=0.0258$. In order to reach $c_{p, \max }$, the system has to be in open circuit condition, thus, no solar cells are present at the bottom of the collector.

In order to derive values for the fraction dependent concentration $c_{p}^{a n}(f)$ analytically, we follow the interpretation of the FC system of Glaeser and Rau [12], Meyer and Markvart [30]. Here, the collection probability $p_{c}$ follows the expression

$$
p_{c}^{*}=\frac{A_{\text {cell }} \Phi_{F C}^{*}}{A_{\text {cell }} \Phi_{F C}^{*}+A_{\text {coll }} \Phi_{\text {out }}^{*}}
$$

with the photon flux absorbed by the solar cells

$$
\Phi_{F C}^{*}=\frac{2 n^{2}}{h^{3} c^{2}} e^{\mu / k T} \int_{E_{1}}^{\infty} E^{2} e^{-E / k T} d E
$$

and the emitted photon flux leaving the collector without hitting a solar cell

$$
\Phi_{\text {out }}^{*}=\frac{2}{h^{3} c^{2}} e^{\mu / k T} \int_{E_{2}}^{\infty} E^{2} e^{-E / k T} d E .
$$

Note, that Markvart proposes an analytical approximation by introducing uniform chemical potentials $\mu$ for all photons in the system. This assumption is exact as long as the system is in open circuit condition. Then the system is in thermal equilibrium and the chemical potential for all photons is equal. This is the same condition for the system Rau et al. [29] describe. As derived in equation (11), the concentration is the ratio between the flux 
L. Prönneke et al.: Geometry effects and loss mechanisms in photovoltaic fluorescent collectors

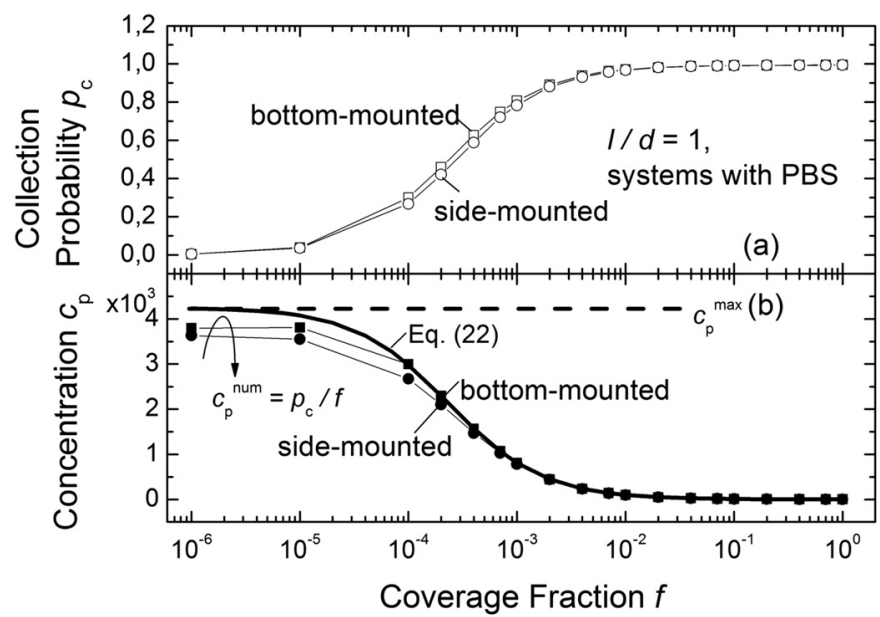

Fig. 6. (a) Collection probabilities $p_{c}$ for photons derived with a Monte-Carlo simulation for a side- and a bottom-mounted system in the statistical limit and with applied PBS. (b) Symbol-lines are the values for the concentration $c_{p}$ derived from $p_{c}$ in (a). Solid lines show the analytically calculated $c_{p}(f)$ from equation (22). Side-mounted system shows less agreement between the analytically and the numerically derived concentration because photons entering the system close to a solar cell are absorbed with a higher probability. This leads to an inhomogeneous chemical potential $\mu$ for the incoming photons which violates the assumptions for the calculation. But, the approach $\mu=$ const. is a good approximation in the statistical limit for the bottom-mounted solar cells [27].

inside the collector $\Phi_{F C}$ and the incident flux $\Phi_{\text {sun }}$. Following the approximately uniform $\mu$ proposed by Markvart et al. and comparing equation (13) to the result of dividing equation (19) by equation (20), we also understand the maximum concentration as

$$
c_{p, \max }^{*}=\frac{\Phi_{F C}^{*}}{\Phi_{\text {out }}^{*}} .
$$

Linking the descriptions of the system via equalizing the photon fluxes in both descriptions with the values derived from the Monte-Carlo simulation $\left(\varphi_{i}^{M C}=\Phi_{i}=\Phi_{i}^{*}\right)$ yields the analytical expression for the concentration

$$
\begin{aligned}
c_{p}^{a n}(f) & =c_{p, \text { max }}=\frac{\varphi_{F C}^{M C}}{\varphi_{\text {sun }}^{M C}}=\frac{N_{\text {coll }}}{A_{\text {cell }}} \frac{A_{\text {coll }}}{N_{\text {in }}}=\frac{p_{c}}{f} \\
& =\frac{1}{f} \frac{A_{\text {cell }} N_{\text {coll }} / A_{\text {cell }}}{A_{\text {cell }} N_{\text {coll }} / A_{\text {cell }}+\left(N_{\text {in }}-N_{\text {coll }}\right)} \\
& =\frac{1}{f} \frac{A_{\text {cell }} \varphi_{F C}^{M C}}{A_{\text {cell }} \varphi_{F C}^{M C}+A_{\text {coll }} \varphi_{\text {out }}^{M C}}=\frac{p_{c}^{*}}{f} \\
& =\frac{A_{\text {coll }}}{A_{\text {cell }}} \frac{A_{\text {cell }} \varphi_{F C}^{M C} / \varphi_{\text {out }}^{M C}}{A_{\text {cell }} \varphi_{F C}^{M C} / \varphi_{\text {out }}^{M C}+A_{\text {coll }} \varphi_{\text {out }}^{M C} / \varphi_{\text {out }}^{M C}} \\
& =\frac{c_{p, \text { max }}^{*}}{c_{p, \text { max }}^{*} f+1}
\end{aligned}
$$

with the coverage fraction $f=A_{\text {cell }} / A_{\text {coll }}$. In Figure $6 \mathrm{~b}$ we derive the numerical concentration $c_{p}^{\text {num }}(f)=p_{c} / f$ from the numerical simulated collection probability $p_{c}$ of Figure $6 \mathrm{a}$ and compare this with the analytically calculated concentration $c_{p}^{a n}(f)$ from equation (22). The analytical solutions excellently fit the statistically derived values for the bottom-mounted system. As described above, the system is described in thermal equilibrium and with same chemical potential $\mu$ for all incoming photons. In particular it holds $q V_{o c}=\mu$ for the cell at the side of the collector. Yet, under short circuit conditions, the voltage $V$ of the cell equals zero and at the solar cell the chemical potential of the photons is $\mu=0$. Therefore, $\mu$ cannot be constant throughout the system. (This finding is also derived by the consideration that in short circuit condition photons enter the system and there they are transported to the solar cells.) A net flux of photons requires local differences in the chemical potential. In a more coarse resolution of the solar cells, at which the period length $l$ exceeds the mean free path of the photons as it is the case for the side-mounted system, the results are not valid any more. Photons entering the system close to a solar cell are absorbed with a higher probability, whereas photons in areas with no solar cell are most likely reabsorbed by the dye and, with a higher probability, reemitted from the collector. Systems in Figures 1a and 1b become identical. Thus, in Figure $6 \mathrm{~b}$ the side-mounted system shows less agreement between the analytically and the numerically derived concentration. However, the approach $\mu=$ constant is a good approximation in the statistical limit for the bottommounted solar cells [27]. We achieve the open-circuit condition by reducing the coverage fraction $f$ of solar cell area to photovoltaic unreasonable low values. The collection probability $p_{c}$ decreases with decreasing $f$, and reduces to nearly zero at $f<10^{-5}$. Concurrently, the concentration approaches the theoretical maximum $c_{p, \max }=4251$. Note, that $c_{p}$ is reaching the maximum $c_{p, \max }$ for coverage fractions $f$ at which $p_{c}$ is almost zero. The thermodynamic limit of the concentration lies therefore beyond photovoltaic useful collector dimensions.

\subsection{Comparison of side-mounted and bottom-mounted FC}

This and the following section compare FCs with bottom-mounted to FCs with side-mounted solar cells. In order to compare the bottom-mounted FC, where the coverage fraction and the collector length are decoupled, with a side-mounted system, we use the modified side-mounted system displayed in Figure 1b. Keeping a constant coverage fraction $f$ upon variation of the collector length $l$ requires the adjustment of the solar cell side length $s$ to

$$
s=f \frac{l^{2}}{4 d},
$$

likewise $s / d=f(l / d)^{2} / 4$ for the normalization of all quantities to the collector thickness $d$. The maximum coverage fraction $f_{\max }$ for the side-mounted system is given by

$$
f_{\max }=\frac{4 d}{l}
$$

because in this case the side length $s$ of the solar cell in equation (23) equals the collector length, and the systems in Figures 1a and 1b become identical. 


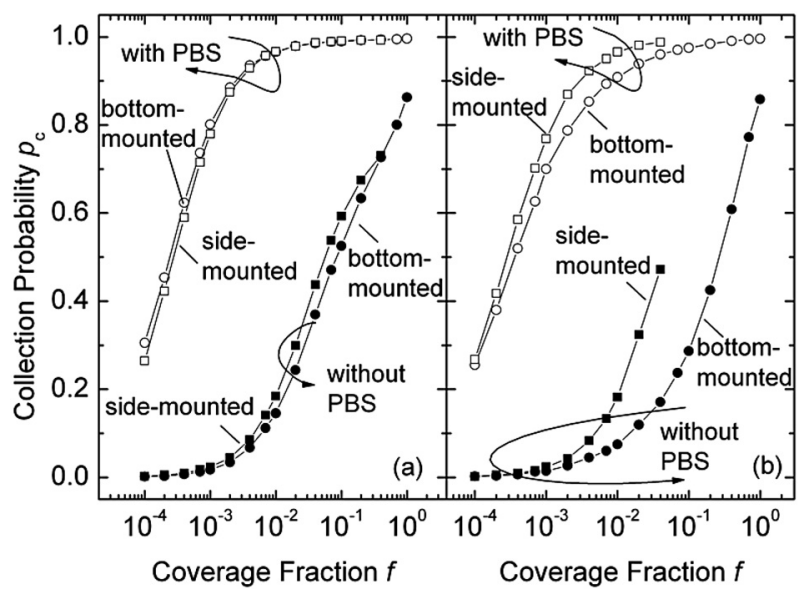

Fig. 7. Comparison of FC systems with solar cells at the sides or at the back as sketched in Figures 1b and 1c. (a) Systems at collector length $l=10 d$. Without PBS side-mounted solar cells provide slightly higher collection probabilities for $10^{-3}<f<4 \times 10^{-1}$. Applying PBS eliminates the difference in collection probability $p_{c}$ for high coverage fractions. Both systems achieve approximately $p_{c}=1$ for coverage fractions $f \approx 1$. For coverage fractions $f<10^{-2}$ mounting solar cells at the FC back side is of slight advantage. (b) Systems at collector length $l=100 d$. Mounting solar cells at collector sides leads to higher collection probabilities for all cases. Compared to Figure 7a, the side-mounted system obtains the same values. Therefore, this system works still in the small-scale limit, whereas for this collector length the bottom-mounted system is already in the transition regime to the large-scale limit which is also shown in Figure 5.

Figures 7a, 7b compare the collection probabilities of side- and bottom-mounted systems for fixed collector length $l=10 d$ (Fig. 7a) and $100 d$ (Fig. 7b). Therefore, for the side-mounted system coverage fractions up to $f_{\max }=0.4$ and 0.04 respectively are modeled.

Figure 7a presents a comparison between bottommounted and side-mounted system, both with a collector length $l=10 d$. The results outline that without applied PBS the side-mounted system performs better for low coverage fractions. The application of PBS on top of the collector yields higher photon collection for the bottom-mounted system in this region. In the region of $f>10^{-2}$ both systems reach collection probabilities close to $100 \%$. As shown in Figure 5 a collector with bottom-mounted solar cells works in the statistical limit. Without the application of a PBS side-mounted solar cells provide slightly higher collection probabilities for $10^{-3}<f<4 \times 10^{-1}$.

Figure $7 \mathrm{~b}$ shows the comparison of the two systems with collector length $l=100 d$. Compared to Figure 7a, the side-mounted system obtains the same values for $p_{c}$. This implies that this system still works in the statistical limit whereas the bottom-mounted system passes into the large-scale region. This is accordingly seen in Figure 5. Therefore, in Figure $7 \mathrm{~b}$ the FC system with solar cells covering the sides performs better at all coverage fractions with or without PBS.

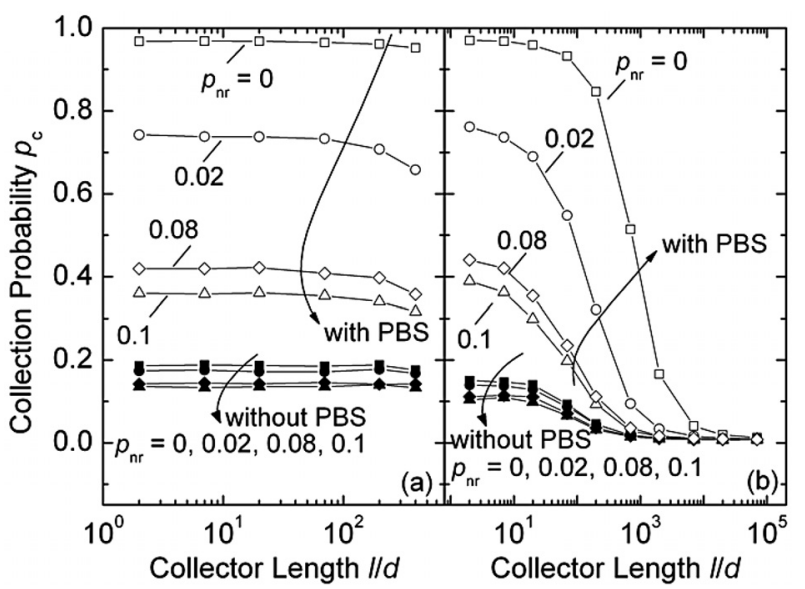

Fig. 8. Comparison of FC systems depicted in Figures 1b and $1 \mathrm{c}$ with a constant coverage fraction $f=0.01$ analyzing influence of non-radiative losses in the dye. (a) Side-mounted system is only modeled up to a collector length $l \leq l_{\max }=4 d / f$ corresponding to a full coverage of all collector sides. Inclusion of non-radiative losses deteriorates the photon collection $p_{c}$ in all cases. The effect is more significant in the system with applied PBS. (b) Bottom-mounted system. The simulation covers a wider range of collector lengths. With PBS the bottommounted system in its radiative limit shows a significant drop in $p_{c}$ for $l / d \geq 100$. The inclusion of non-radiative losses reduces this limit to $l / d \geq 1$. In comparison: for $p_{n r}=0$ (only radiative losses) the side-mounted system leads to a better photon collection $p_{c}$, especially at large values of $l$. Inclusion of non-radiative losses leads to the same behavior in the largescale region, but for collector lengths in the statistical limit, the bottom-mounted system performs better. Without PBS the side-mounted system yields higher $p_{c}$ at all collector lengths even with inclusion of non-radiative losses.

\subsection{Loss mechanisms}

This section analyzes the systems in the radiative limit as well as the influence of non-radiative losses in the dye, of a non-perfect mirror at the back side, and of a non-ideal reflection cone at the PBS filter. Unlike in Figures 7a, 7b, we now vary the collector length $l / d$ while assuming a constant coverage fraction $f=0.01$ for all simulations and an additional $f=0.9$ for Figures $9 \mathrm{a}, 9 \mathrm{~b}$. Thus, the side-mounted system is modeled up to a maximum collector length $l=l_{\max }=4 d / f=400 d$ and $4.44 d$, respectively, where the side length $s$ of the solar cells equals the collector length $l$.

\subsubsection{Non-radiative losses in the dye}

Figures $8 \mathrm{a}, 8 \mathrm{~b}$ compare the side- and the bottommounted systems in their radiative limit with a probability $p_{n r}=0$ for non-radiative losses and under the influence of non-radiative recombination in the dye $\left(p_{n r}=0.02,0.08\right.$ and 0.1 ). Figure $8 \mathrm{a}$ shows the results for the side-mounted system. In the radiative limit the application of a PBS increases the photon collection from $p_{c} \approx 19 \%$ to $p_{c} \approx 95 \%$. These values remain constant up to the maximal simulated collector length $l_{\max }=400 d$. As discussed above, 


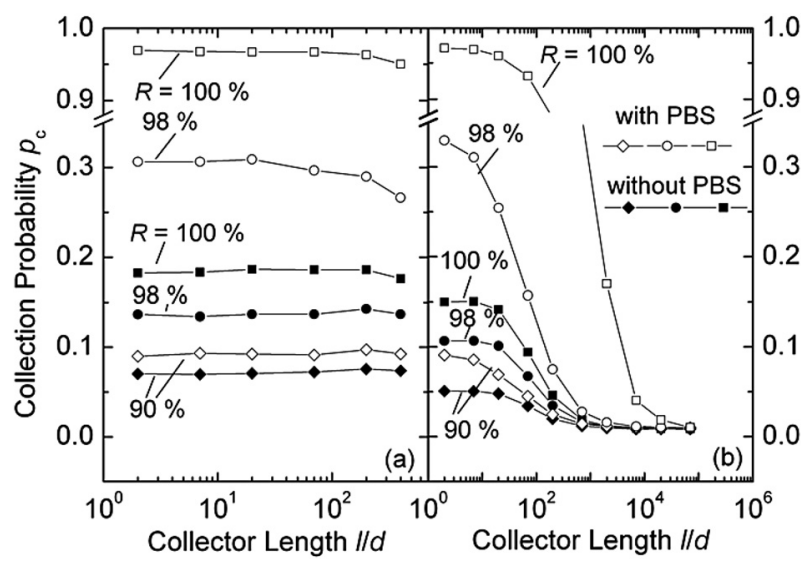

Fig. 9. Influence of non-perfect mirror at the back side of the FC in systems with a coverage fraction $f=0.01$. (a) Side-mounted solar cells. Inclusion of non-radiative losses in the dye decreases the photon collection significantly. The relative deterioration in a system with applied PBS is higher than in the system without PBS. (b) Bottom-mounted solar cells. At small $l / d$ the system shows a similar behavior as the sidemounted system.

this means that the re-absorption length in this system lies considerably under the collector length.

Figure $8 \mathrm{~b}$ presents the results for the bottom-mounted system. Note, that the simulation covers a larger range of collector lengths $l / d$. As explained above, enlarging the systems degrades $p_{c}$ even more than the inclusion of nonradiative recombination in the dye. However, the lowering in the photon collection occurs with $p_{n r}=0$ at $l / d=100$, but this limit reduces to $l / d \leq 10$ for $p_{n r}=0.1$.

Under the application of PBS in the radiative limit both systems display a similar $p_{c} \approx 96.5 \%$ in the limit of small collector lengths $l / d<10$. Whereas the data for the bottom-mounted system considerably decay at $l / d>100$, a high collection probability of $p_{c}>95 \%$ is maintained by the side-mounted system up to the maximum collector length $l_{\max } / d=400$. The inclusion of non-radiative losses in the calculation deteriorates the collection probability in both cases significantly. Also, the transition to large-scale limit occurs at lower collection lengths. Interestingly, in the small-scale limit $l / d<10$ the bottom-mounted system achieves a better photon collection than the sidemounted system. Here, the collection probability decreases by $\Delta p_{c} \approx 52 \%$ from $p_{c} \approx 98 \%$ to $p_{c} \approx 46 \%$ whereas in the side-mounted system it decreases by $\Delta p_{c} \approx 55 \%$.

Comparing these two systems without the application of PBS shows that the side-mounted option displays a collection probability that is consistently higher than that of the bottom mounted systems. In addition, the transition towards the large-scale limit that occurs at $l / d \approx 20$ for the cells at the bottom is absent for the side mounted system. A collection probability $p_{c}>18 \%$ is maintained up to $l_{\max }$. With included non-radiative loss $p_{n r}=0.08$ the over-all collection probability drops by about $3 \%$ for both options. The difference between side- and bottommounted systems remains the same.

\subsubsection{Reflection losses at the back side mirror}

Mirrors cover the collector back sides either fully in the side-mounted system or partly in the bottom-mounted system. Optical losses due to non-ideal reflection are a major loss mechanism especially for large collector systems. Note that other losses, especially parasitic absorption in the polymer matrix (not considered in the present paper), are expected to have comparable effects. In the following, we study the influence of reflection coefficients $R<1$ in comparison to the perfect case $(R=1)$. We do not consider explicitly the case of an air-gap between mirror and collector which would restrict the losses to angles larger than the critical angle of total internal reflection. The present approach uses $R$-values which are independent of the direction of the photons and could be looked at as directional averages.

Figures $9 \mathrm{a}, 9 \mathrm{~b}$ depict the influences of reflection losses on the photon collection $p_{c}$ of both systems. Figure 9a presents the results for the side-mounted system. Since less radiative losses occur in the system with PBS the contribution of non-radiative reflection losses at the back side mirror is more significant. Therefore, the decrease in the photon collection is higher.

Figure $9 \mathrm{~b}$ shows the photon collections for the system with bottom-mounted solar cells. For small $l / d$ the system behaves similar to the side-mounted system. The larger the system the more often a reflection takes place, thus, increasing the number of lost photons. Therefore, included reflection losses decrease the photon collection already at $l / d=10$, whereas for a mirror with $R=1$ this drop occurs at $l / d=100$.

In systems with coverage fraction $f=0.01$ at any of the calculated collector length the re-absorption is a rare event compared to the number of reflections at the FC back side as the following example clarifies. Most photons emitted by the dye experience the absorption coefficient $\alpha_{2}=0.03 / d$ as mentioned above. With $d=3 \mathrm{~cm}$, a typical thickness for industrialized fluorescent collectors, the photons are re-absorbed after $100 \mathrm{~cm}$. A photon emitted with an angle $\theta=45^{\circ}$ hits the collector sides every $4.2 \mathrm{~cm}$. Since the coverage fraction $f=0.01$ is very low, most likely the photon is re-absorbed before it hits a solar cell. Such, a photon with $\theta=45^{\circ}$ is reflected 23 times before it is reabsorbed. Therefore, a non-perfect mirror has a stronger influence than an equally high non-radiative recombination loss in the dye. A reflection $R=98 \%$ at the back side mirror causes a drop $\Delta p_{c} \approx 70 \%$ for small $l / d$ which is higher than the drop $\Delta p_{c} \approx 25 \%$ caused by the nonradiative recombination loss as shown in Figures 8a, 8b. Therefore, the mirror applied in the system has to feature a superior quality compared to the fluorescent dye.

\subsubsection{Non-perfect photonic structure}

In the simulations the application of a photonic band stop as an energy selective filter increases the photon collection in all cases. However, in realistic filters blocking the photons depends not only on the energy but also on the angle of incidence of the photon as schematically shown in 


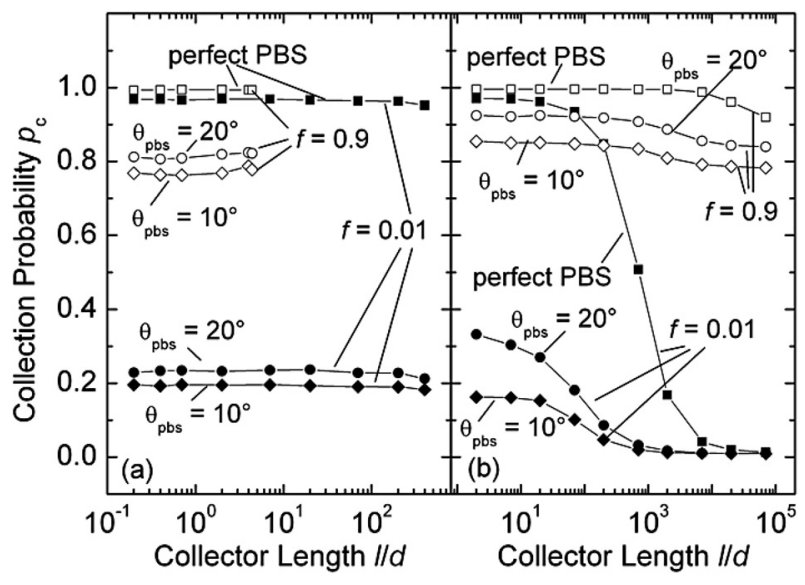

Fig. 10. Influence of a photonic band stop filter with angular selectivity. (a) Side-mounted system. Coverage fraction $f=0.9$ achieves higher photon collections $p_{c}$ than $f=0.01$ for all $\theta_{p b s}$. (b) Bottom-mounted system. For small scales the system shows a better performance than the side-mounted system because this system benefits from the randomization of photon direction during re-emission.

Figure 3e. Figures 10a, 10b outline the influence of smaller reflection cones $\left(\theta_{p b s}=10^{\circ}\right.$ and $\left.20^{\circ}\right)$ on side- and bottommounted systems with coverage fractions $f=0.01$ and 0.9 .

Figure 10a depicts the results for the side-mounted system. The application of a non-perfect PBS filter decreases the photon collection at all collector lengths. However, a relatively higher drop occurs for the system with $f=0.01$ than for the system with $f=0.9$. Here, a higher re-absorption rate occurs due to longer distances between the cells. Therefore, photons are more often re-emitted with their direction spatially randomized. The frequent randomization carried out also in unfavorable angles contributes to the number of lost photons.

Figure 10b presents the calculation results for the bottom-mounted system. At small $l / d$ this system collects more photons under the application of a non-perfect PBS filter than the side-mounted system. This is due to the effect that the disadvantageous angles for the PBS are very favorable angles for solar cells at the FC back side. Therefore, the collection of a photon is more likely than in the side-mounted system. As in the side-mounted system, the collection in the system with $f=0.01$ decreases more at large $l / d$ than in the system with $f=0.9$.

\subsubsection{Realistic values}

In order to get a more realistic picture, we perform a system simulation which includes several loss mechanisms simultaneously. While the absorption/emission in the fluorescent collector is as ideal as presented in Figure 2, the non-radiative recombination rate lies at $5 \%$, which is a value typically achieved in industrial FCs. For the back side mirror, we assume a reflectivity $R=92 \%$, which corresponds to the reflectivity of Aluminium. Furthermore, we assume dielectric layers as photonic structures which show transmittances $T>95 \%$ if manufactured carefully.

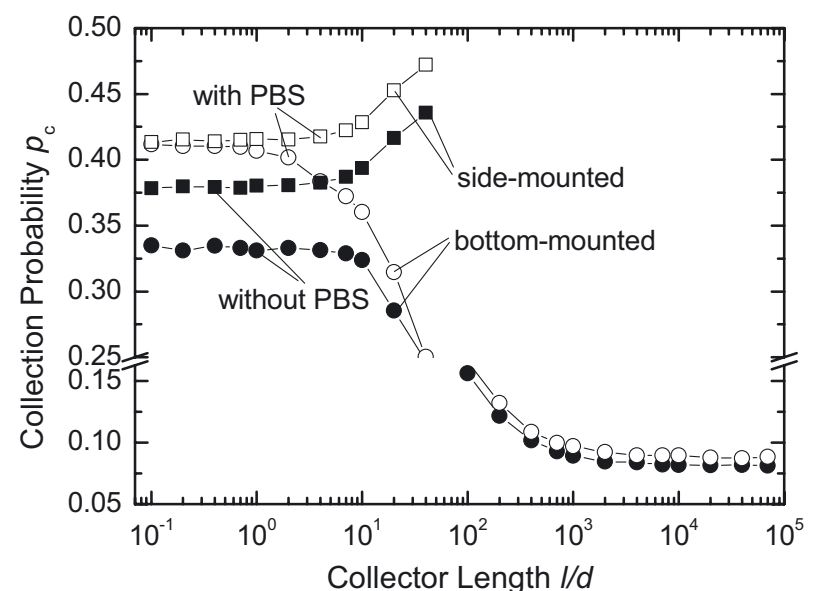

Fig. 11. Side-mounted and bottom-mounted solar cells on fluorescent collectors including realistic values for the back side mirror, the photonic structure and the fluorescent dye. The bottom-mounted solar cells are in the small-scale limit for $l / d<1$ and in the large-scale limit from $l / d>10^{3}$. For a clear acrylic glass on top of the solar cells, the coverage fraction $f=0.1$ leads to a collection probability $p_{c}=0.1$. Thus, a fluorescent collector system with the assumed losses increases the collection probability by $23 \%$, with a PBS on top by $27 \%$. The side-mounted solar cells show an interesting behavior for $l / d>11$. Instead of descending into the large-scale limit, $p_{c}$ increases up to 0.47 at $l / d>40$ (with PBS on top).

However, they always show a blue shift in their transmittance spectrum for oblique incident angles. Thus, adjusting the transmittance spectrum properly should lead to a full band stop for all ray directions. Nevertheless, we assume $\theta_{p b s}=30^{\circ}$. The coverage fraction is $f=0.1$. Note, that for a clear acrylic glass on top of the solar cells, the coverage fraction $f=0.1$ leads to collection probability $p_{c}=0.1$.

Figure 11 shows that bottom-mounted solar cells perform as expected from the previous results: the system is in the small-scale limit for $l / d<1$ and in the large-scale limit from $l / d>10^{3}$. Thus, a FC system with the assumed losses increases the collection probability by $23 \%$, with a PBS on top even by $27 \%$ compared to clear acrylic glass. The side-mounted solar cells show an interesting behavior for $l / d>11$. Instead of descending into the large-scale limit, $p_{c}$ increases up to 0.47 at $l / d>40$ (with PBS on top). We explain this effect, which also is already slightly indicated in the data of Figure 10a, as follows: the larger $l / d$, the more collector edge area is covered with solar cells until they are fully covered for $l / d=40$. Thus, the photon path length is larger for small $l / d$ than for large $l / d$, because the probability to hit solar cell area is smaller. The longer the path length, the higher is the probability for the photon to be subject to loss mechanisms.

\section{Conclusion}

Monte-Carlo simulations compare photovoltaic fluorescent collectors in side-mounted and bottom-mounted systems as well as the systems with or without the application 
of a photonic band stop filter on top. The filter greatly enhances the overall collection probability by suppressing emission of converted photons from the surface of the collector. Also, the sensitivity of the collector to repeated reabsorption and re-randomization of the fluorescent photons is reduced. We find that the collection probability of systems with identical coverage fraction, i.e., the same amount of solar cell area per unit collector area is heavily influenced by the scaling of the solar cell size. Many small solar cells generally perform better than few large solar cells with the same overall area (scaling-effect). The systems in their radiative limits are compared to systems which include loss mechanisms. The comparison of collectors with solar cells mounted at the collector side to a system with the cells at the bottom shows that in most cases the side-mounted system displays a higher collection efficiency. We find that a non-perfect reflection at the back side mirror causes a higher deterioration in the photon collection than a comparable non-radiative recombination in the dye. This is because in the analyzed systems the reabsorption is a rare event compared to a reflection at the back side. Therefore, the quality of the back side mirror merits especial care. Assuming a restricted reflection cone of the photonic band stop filter causes higher losses in the side-mounted system than in the bottom-mounted system which is caused by the randomization of the photon angles during the emission by the dye. All loss effects are especially significant for systems without a filter. We see that a good quality of the filter is especially necessary in systems with low coverage fraction of solar cells. In comparison to the classical side-mounted system, the system with solar cells at the back side performs equally well for small scales. Therefore, applying fluorescent collectors technically less expensive on top of solar cells is a promising approach, if the solar cells are properly scaled in size and distance.

This work was supported by a grant of the Deutsche Forschungsgemeinschaft (DFG, contract PAK88, 'nanosun'). The authors wish to thank G. Bilger, C. Ulbrich, and T. Kirchartz for numerous discussions as well as J.H. Werner for continuous support.

\section{References}

1. A. Goetzberger, V. Wittwer, Sol. Cells 4, 3 (1981)

2. A. Goetzberger, W. Greubel, Appl. Phys. A 14, 123 (1977)

3. T. Trupke, M.A. Green, P. Würfel, J. Appl. Phys. 92, 4117 (2002)
4. T. Trupke, M.A. Green, P. Würfel, J. Appl. Phys. 92, 1668 (2002)

5. J.A.M. van Roosmalen, Semiconductors 38, 970 (2004)

6. A.J. Chatten, K.W.J. Barnham, B.F. Buxton, N.J. EkinsDaukes, M.A. Malik, Solar Energy Mater. Solar Cells 75, $363(2003)$

7. E. Yablonovitch, J. Opt. Soc. Am. 70, 1362 (1980)

8. G. Smestad, H. Ries, R. Winston, E. Yablonovitch, Solar Energy Mater. Solar Cells 21, 99 (1990)

9. L.H. Slooff, E.E. Bende, A.R. Burgers, T. Budel, M. Pravettoni, R.P. Kenny, E.D. Dunlop, A. Büchtemann, Phys. Stat. Sol. PRL 2, 257 (2008)

10. M. Bendig, J. Hanika, H. Dammertz, J.C. Goldschmidt, M. Peters, M. Weber, in IEEE/EG Symposium on Interactive Ray Tracing, California, USA, 2008, p. 93

11. U. Rau, F. Einsele, G.C. Glaeser, Appl. Phys. Lett. 87, 171101 (2005)

12. G.C. Glaeser, U. Rau, in Proc. SPIE, edited by A. Gombert (2006), Vol. 6197, p. 143

13. J.C. Goldschmidt, M. Peters, L. Prönneke, L. Steidl, R. Zentel, B. Bläsi, A. Gombert, S. Glunz, G. Willeke, U. Rau, Phys. Stat. Sol. A 205, 2811 (2008)

14. M. Peters, J.C. Goldschmidt, P. Löper, B. Bläsi, A. Gombert, J. Appl. Phys. 105, 014909 (2009)

15. S. Knabe, N. Soleimani, T. Markvart, G.H. Bauer, Phys. Stat. Sol. PRL 4, 118 (2010)

16. J.C. Goldschmidt, M. Peters, A. Bösch, H. Helmers, F. Dimroth, S.W. Glunz, G. Willeke, Solar Energy Mater. Solar Cells 93, 176 (2009)

17. R. Reisfeld, Opt. Mater. 32, 850 (2010)

18. L. Prönneke, G.C. Glaeser, Y. Uslu, U. Rau, in 24th European Photovoltaic Solar Energy Conference Hamburg, Germany, 2009, p. 385-387

19. T. Markvart, J. Appl. Phys. 99, 026101 (2006)

20. E. Yablonovitch, Phys. Rev. Lett. 58, 2059 (1987)

21. E. Yablonovitch, J. Opt. Soc. Am. B 10, 283 (1993)

22. E. Yablonovitch, Opt. Lett. 23, 1648 (1998)

23. L. Martinu, D. Poitras, J. Vac. Sci. Technol. A 18, 2619 (2000)

24. D.N. Chigrin, C.M. Sotomayor Torres, Opt. Spectrosc. 91, $484(2001)$

25. M. Schöfthaler, U. Rau, J.H. Werner, J. Appl. Phys. 76, 4168 (1994)

26. P. Kittidachan, L. Danos, T.J.J. Meyer, N. Aldermann, T. Markvart, Chimia 61, 780 (2007)

27. G.C. Gläser, Ph.D. thesis, University of Stuttgart, 2007

28. T. Markvart, Appl. Phys. Lett. 88, 176101 (2006)

29. U. Rau, F. Einsele, G.C. Glaeser, Appl. Phys. Lett. 88, $176102(2006)$

30. T.J.J. Meyer, T. Markvart, Appl. Phys. 105, 063110 (2009) 